ISSN 1870-4069

\title{
Heuristic Mechanism for Drone Swarm Auto-Organization
}

\author{
Juan Muñoz, Alberto Ochoa, Israel Hernández, Gilberto Rivera, Felipe Vázquez
}

Universidad Autónoma de Ciudad Juárez, Chihuahua, Mexico

alberto.ochoa@uacj.mx

\begin{abstract}
Current technology used for fires are: meteorological stations and satellite image and satellites. This last is a great option, but, from space, the fire detection is until it is large enough to be seen from orbital altitude. A drone can be used to monitor a forest looking for fire signs before the satellite observes it, but a single drone to cover a large acre surface is not optimal. A drone swarm with auto-organization capacity, equipped with atmospheric sensors that detect fire hazard conditions or even a fire in an early stage, needs to be used to optimize the area coverage. Implement a heuristic algorithm for drone swarm autoorganization applicable for wildfire alert and detection. Forest fires are a big environmental problem due they are mainly detected until they have burned some square kilometers. When these are detected at the developed stage, the fire will be difficult to contain. Some wildfires affect agricultural along as residential areas causing significant economic loses.
\end{abstract}

Keywords: wildfires prevention, heuristic algorithms.

\section{Introduction}

\subsection{Background}

According to Bala et al [1], the prevention of deforestation and promotion of afforestation have often been cited as strategies to slow global warming. Deforestation releases $\mathrm{CO} 2$ to the atmosphere, which exerts a warming influence on Earth's climate. However, biophysical effects of deforestation, which include changes in land surface albedo, evapotranspiration, and cloud cover also affect climate.

Deforestation has several causes, but wildfires and illegal tree cut, which are manmade, are the mayor. Current technologies used to detect wildfires are: meteorological stations and satellite image and satellites. This last is a great option, but, from space, 
the fire detection is until it is large enough to be seen from orbital altitude. A drone can be used to monitor a forest looking for fire signs before the satellite observes it, but a single drone to cover a large acre surface is not optimal.

A drone swarm with auto-organization capacity, equipped with atmospheric sensors that detect fire hazard conditions or even a fire in an early stage, needs to be used to optimize the area coverage. Implement a heuristic algorithm for drone swarm autoorganization applicable for wildfire alert and detection. Forest fires are a big environmental problem due they are mainly detected until they have burned some square kilometers. When these are detected at the developed stage, the fire will be difficult to contain. Some wildfires affect agricultural along as residential areas causing significant economic loses. Unmanned Aerial Vehicle (UAV) is mainly known as drone. These devices have been flying for some decades now, mainly for military purposes. From 2010 to date, these have been more accessible for non-military purposes. Photography, real estate, utilities and construction are the main fields that have adapted these technologies.

\section{Forest Fire Prevention Using Data-Logging/Transmitting Drone Swarm Triad}

\subsection{Forest Wild Fires in Chihuahua State}

Forest fires are one of the main causes of forest loss. Vegetation covers in forest ecosystems and as a consequence erosion and soil degradation. Historically, our State has seen affected by this type of casualties, the years of greatest occurrence were in the 2011 and 2012, which showed a significant decrease to the year 2015, returning to present a rebound during the 2016, with a historical average of 870 annual fires between 1995 and 2016.

The main causes of fires are still those related to Agricultural activities, slash and burn and the Crops, with a percentage that fluctuates between 25 and $60 \%$. Regarding the type of ecosystem (CONAFOR 2015), the most affected is the

Cold temperate climate, followed by natural pasture. The smaller area

Report is the arid and semi-arid, as for forests there were no fires. The most common type of fire is the superficial fire.

The year 2015 presented totally atypical conditions, since only Presented 252 forest fires, affecting a total area of 1,974.05 ha. The municipalities that historically have the largest affected area are Guadeloupe and Bald, Wood, Bocoyna, Guachochi and Balleza. The municipalities less affected were Janos, Casas Grandes, Ocampo and Urique [2].

Table 1. Historical data of wild fires in Chihuahua, Mexico.

\begin{tabular}{lccc}
\hline YEAR & $\begin{array}{c}\text { QUANTITY } \\
\text { OF FIRES }\end{array}$ & $\begin{array}{c}\text { BURNED } \\
\text { SURFACE }\end{array}$ & $\begin{array}{c}\text { AVERAGE } \\
\text { SURFACE }\end{array}$ \\
\hline 2006 & 1,057 & 18,505 & 17.51 \\
\hline
\end{tabular}


Heuristic Mechanism for Drone Swarm Auto-organization

\begin{tabular}{lccc}
\hline YEAR & $\begin{array}{c}\text { QUANTITY } \\
\text { OF FIRES }\end{array}$ & $\begin{array}{c}\text { BURNED } \\
\text { SURFACE }\end{array}$ & $\begin{array}{c}\text { AVERAGE } \\
\text { SURFACE }\end{array}$ \\
\hline 2007 & 625 & 10,560 & 16.9 \\
\hline 2008 & 1,153 & 17,216 & 14.93 \\
\hline 2009 & 842 & 10,704 & 12.71 \\
\hline 2010 & 697 & 29,316 & 42.06 \\
\hline 2011 & 1,687 & 87,920 & 52.12 \\
\hline 2012 & 1,473 & 55,979 & 37.47 \\
\hline 2013 & 1,137 & 30,554 & 26.87 \\
\hline 2014 & 817 & 17,600 & 21.52 \\
\hline 2015 & 251 & 1,974 & 7.83 \\
\hline 2016 & 701 & 13,353 & 19.04 \\
\hline AVERAGE & $\mathbf{9 4 9}$ & $\mathbf{2 6 , 6 9 8}$ & $\mathbf{2 4}$ \\
\hline
\end{tabular}

\section{Problem Formulation}

As stated above, forest fires are a problem due the devastation it leaves, environmental and economic. Big efforts are applied to solve the wildfire, but due the large extensions of the forests, most are detected at a late state that commonly leads to large spread with high difficulty to contain.

Surface recognition is a very common need like for agriculture, disaster relief operations, goods delivery, etc. Depending on the area size, this could take a significant amount of time.

For this, there is a need to use tools like a drone, but, also, this need so to have a mechanism to efficiently perform these activities and, mainly, surface recognition to optimize time and resources. Figure 2 depicts need to coordinate the drone swarm.

\subsection{Formalization of the Problem}

We consider the problem of monitoring a large geographical area using a drone swarm to prevent forest fires. The area to be monitored is divided into well identified subareas. A drone swarm is composed by a set of heterogeneous drones, which are located at a starting point.

Thus, the problem that we tackle is to create a schedule containing the assignment of drones to geographical sub-areas for monitoring and detecting forest fires, such that the schedule completion time is minimized. In figure 1 , the geographical area is represented in quadrants to define the tasks surface. 


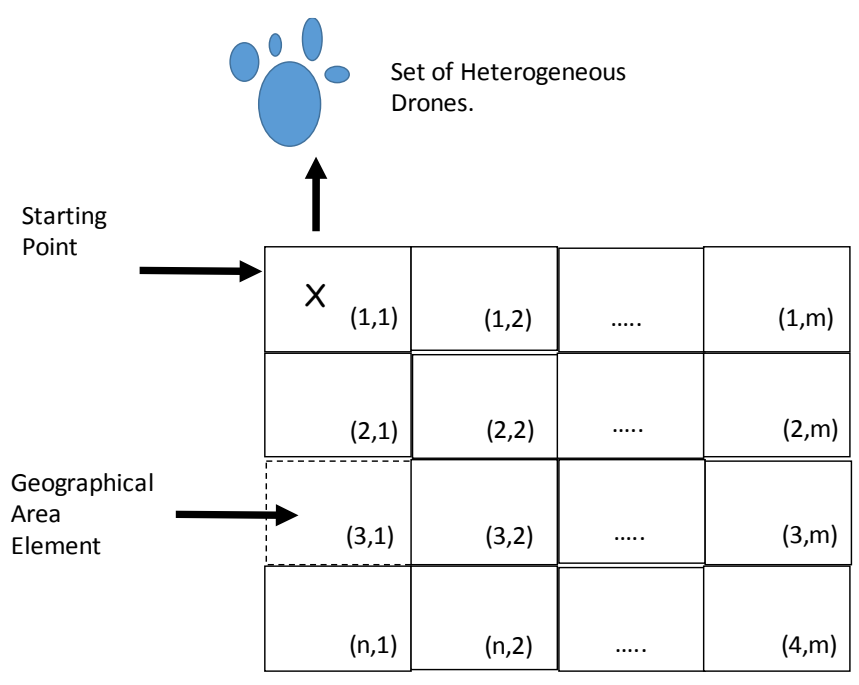

Fig. 1. Representation of the surface.

A scheduling system model for planning the visit of drones to geographical subareas consists of the following elements: the geographical area, drones swarm and an objective function for scheduling.

\subsection{Large Geographical Area}

The geographical area is denoted by $A$. Without a loss of generality, we assume that A has a square shaped area and does not contain any obstacle. The square shape was chosen for simplicity in the model. The area $A$ can be divided into finite sub-areas forming a vector $A=\left\{a_{0}, a_{1}, a_{2}, . . a_{n m}\right\}$ of dimension n-by-m. For convenience, we consider $\mathrm{a}_{0}$ as the base from which drones depart and return after complete their mission. We use Geo $\left(\mathrm{a}_{\mathrm{i}}\right)$ to denote the geographical position of $\mathrm{a}_{\mathrm{i}}$ on $\mathrm{A}$.

\subsection{Heterogeneous Drone Swarm}

The Heterogeneous Drone Swarm (HDS) can be represented by a DAG HDS::(D, E). $D$ represents the set of heterogeneous drones that compose the swarm. $E$ is the set of directed arcs connecting different pairs of drones, so $e\left(d_{i}, d_{j}\right)$ denotes a precedence that indicates that dron $d_{j}$ cannot start its mission until $d_{i}$ finishes its mission. For convenience, Pred $\left(d_{i}\right)$ denotes the subset of drones that directly precede $d_{i}$ and Succ $\left(d_{i}\right)$ denotes the subset of drones that directly follow $d_{i}$.

The entry drone are those with $\mid$ Pred $\left(d_{i}\right) \mid=0$ and the output drone are those with $\left|\operatorname{Succ}\left(d_{i}\right)\right|=0$. For simplicity, in these cases we consider the use of dummy tasks such that the dag contains only one entry and output dron. Remembering that the drones are heterogeneous, we represent the estimated flying time from the base at a $a_{0}$ with EFT :D $\times A \rightarrow \operatorname{Int}$, where $\operatorname{EFT}\left(d_{i}, a_{j}\right)$ denotes the time for a dron $d_{i}$ to reach a geographical sub- 
area $\mathrm{a}_{\mathrm{j}}$. For simplicity, we consider that the flying time to return to the base at $\mathrm{a}_{0}$ is the same than the time to reach a particular area from $\mathrm{a}_{0}$. A dron can be assigned to different missions, but it can only perform one mission at time. Thus, at time $t$ we consider avail $: D \rightarrow[0 . .1]$, which captures the availability of each dron at time $t$. Note that the time of the mission of a particular dron is given when it is working at full availability. $W\left(d_{i}\right)$ denotes the time for a dron $d_{i}$ to execute certain work once it reaches a geographical sub-area. Setup $\left(d_{i}\right)$ denotes the setup time for a dron to start a new mission. We assume that information about the flying and setup time are provided in standard time units, compatible with our drone performance measures.

\subsection{Scheduling Problem}

Scheduling drones to geographical areas requires the consideration of four events: (a) the time at which the dron starts its mission. (b) The time for a dron to reach a particular geographical area. (c) The time for a dron to perform certain work once it reaches its geographical area and (d) the time for a dron to return to the base.

Thus, we first need to predict the time at which a particular dron departs from $a_{0}$ to perform its mission to a particular sub-area and the time in which the dron returns to the base. We must first define two mutually referential quantities. $\operatorname{EDT}\left(d_{i}, a_{m}\right)$ is the Estimated Departing Time of dron $d_{i}$ to $a_{m}$, it is calculated by:

$$
\operatorname{EDT}\left(d_{i}, a_{m}\right)=\operatorname{Setup}\left(d_{i}\right)+\max d_{j} \in \operatorname{Pred}(\operatorname{di})\left\{\operatorname{ERT}\left(d_{j}, a_{0}\right)\right\} .
$$

$\operatorname{Setup}\left(d_{i}\right)$ is preparation time for a dron to start a new mission. It is added to the result of the max block in Equation (3), which returns the maximum estimated returning time in which each drones in $\operatorname{Pred}\left(d_{i}\right)$ return to the base. This is calculated by $\operatorname{ERT}\left(d_{i}, a_{0}\right)$, which denotes the Estimated Returning Time of dron $d_{i}$ to the base located at $\mathrm{a}_{0}$ and it is calculated by:

$$
\operatorname{ERT}\left(d_{j}, a_{0}\right)=\operatorname{EDT}\left(d_{j}, a_{m}\right)+\left(2 * \operatorname{EFT}\left(d_{j}, a_{m}\right)\right)+W\left(d_{j}\right) .
$$

Once that all the drones have been scheduled, the estimated completion time of the schedule is determined by the estimated return time of the output dron. The estimated completion time is also known as the schedule make span:

$$
\operatorname{ERT}\left(d_{\text {output }}, a_{m . n}\right) \text {. }
$$

The objective function for drone scheduling aims to create a schedule containing the assignment of drones to geographical sub-areas such that its make span is minimized.

\subsection{DERT Algorithm}

The DERT algorithm is based on the well-known list scheduling approach. Our interest in this approach is to explore low computational complexity strategies and apply them to prevent and combat forest fires with the use of drones. Thus, the DERT algorithm basically consists of two phases: The drone prioritization phase in which a priority rank assignment is set to each dron. The geographical sub-area assignation phase where 
each dron is assigned to that geographical sub-area which optimizes a predefined cost function. The DERT algorithm is shown in Figure 2.

\section{Drone Prioritization Phase}

We use $D R u\left(d_{i}\right)$, an upward rank defined as the length of the critical path from dron $d_{\mathrm{i}}$ to the output dron. $D R u\left(d_{i}\right)$ is calculated recursively as:

$$
\operatorname{DRu}\left(d_{i}\right)=\operatorname{avg}\left(F T_{i}\right)+\max v_{j} \in \operatorname{Succ}(v i)\left(\operatorname{DRu}\left(v_{j}\right)\right),
$$

where $\operatorname{avg}\left(F T_{i}\right)$ is the average of the visit time for a dron $d_{i}$ across all sub-areas:

$$
\operatorname{avg}(F T(d i))=\sum_{k=0}^{n m} \frac{(d i, a k)}{n \cdot m} .
$$

1. Set the drone flying time.

2. Set the drone setup time.

3. Set the drone work time.

4. Calculate $D R_{u}$ for each dron by traversing the graph from the exit node to the entry node and keep the values in $L$.

5. Sort the drones in $L$ in descending order of $D R_{u}$ values.

6. Create a list $L S A$ with the sub-areas composing A.

7. while there are unvisited areas in $\boldsymbol{L S A}$ do

8. Select the first sub-area $a_{m}$ from $L S A$

9. for each available dron $d_{i}\left(\operatorname{avail}\left(d_{i}\right)=1\right)$ in $\mathrm{L}$ do

10. Compute $\operatorname{EDT}\left(d_{i}, a_{m}\right)$ value.

11. Compute $E R T\left(d_{i}, a_{0}\right)$ value.

12. Assign dron $d_{i}$ to the sub-area $a_{m}$ that minimizes $E R T$ of $d_{i}$

13. Set avail $\left(d_{i}\right)=0$ from the time between EDT and ERT.

14. end while

Fig. 2. DERT algorithm.

\section{Sub-area Assignation Phase}

The DERT algorithm considers that a dron can be assigned to several missions, but it only can perform once at time. A mission involves to depart from the base $a_{0}$ to an assigned area $\mathrm{a}_{\mathrm{m}}$, perform a work once it reaches $a_{m}$ and return to $\mathrm{a}_{0}$. In our case, the work that a dron performs at a particular area is to monitor. The assignation phase where a dron is assigned to a geographical sub-area offering the minimum estimated returning time, takes $O(d x e)$ time complexity for $d$ drones a $e$ precedencies. 


\subsection{Example}

Table 2. Sub-area assignation matrix.

\begin{tabular}{|c||c|c|c|c|c|c|c|c|c|c|c|c|}
\hline Dron & $\mathrm{a} 1$ & $\mathrm{a} 2$ & $\mathrm{a} 3$ & $\mathrm{a} 4$ & $\mathrm{a} 5$ & $\mathrm{a} 6$ & $\mathrm{a} 7$ & $\mathrm{a} 8$ & $\mathrm{a} 9$ & $\mathrm{a} 10$ & $\mathrm{a} 11$ & 12 \\
\hline 1 & 7 & 3 & 9 & 4 & 9 & 4 & 3 & 3 & 1 & 2 & 9 & 9 \\
\hline 2 & 4 & 7 & 18 & 3 & 2 & 7 & 7 & 2 & 7 & 8 & 7 & 5 \\
\hline 3 & 8 & 2 & 19 & 4 & 7 & 1 & 2 & 8 & 4 & 4 & 4 & 1 \\
\hline 4 & 9 & 9 & 17 & 3 & 2 & 7 & 9 & 1 & 2 & 2 & 3 & 5 \\
\hline 5 & 4 & 8 & 10 & 5 & 9 & 9 & 8 & 8 & 9 & 1 & 4 & 9 \\
\hline 6 & 5 & 5 & 9 & 8 & 2 & 3 & 5 & 4 & 5 & 8 & 8 & 4 \\
\hline 7 & 2 & 3 & 11 & 2 & 3 & 3 & 3 & 7 & 3 & 2 & 7 & 3 \\
\hline 8 & 7 & 5 & 14 & 4 & 7 & 5 & 5 & 5 & 5 & 3 & 3 & 4 \\
\hline 9 & 3 & 4 & 20 & 3 & 4 & 4 & 4 & 4 & 2 & 4 & 1 & 4 \\
\hline 10 & 1 & 5 & 2 & 7 & 2 & 5 & 5 & 5 & 10 & 7 & 3 & 2 \\
\hline
\end{tabular}

\section{$4 \quad$ Future work}

Overview. In this section, the future work for the drone swarm algorithm is described, the next challenges to be taken and opportunities.

\subsection{Fire Propagation Simulation Using Support Vector Machine}

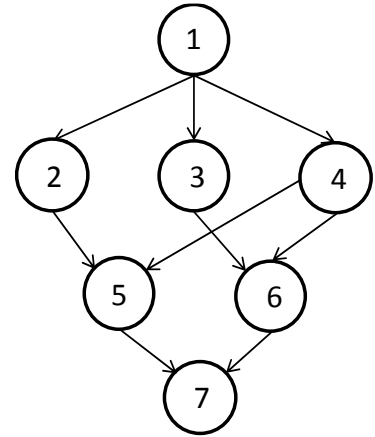

(a) Drone Swarm

\begin{tabular}{|c|c|c|c|}
\hline a1 & a2 & a3 & a4 \\
\hline \hline a5 & a6 & a7 & a8 \\
\hline \hline a9 & a10 & a11 & a12 \\
\hline
\end{tabular}

(b) Area to explore

Support vector machine (svm) will be used to analyze a given dataset from the forest fires described in this work. The objective of svm is to have a tool so forest fires could be characterized and serve as base for propagation prediction, that lead to adapting the drone swarm algorithm to search for fire spots in the early stages of wild fires so its vicious propagation could be prevented. 


\subsection{Illegal Tree-cutting Detection}

Another opportunity detected is that, all around the world, illegal tree cutting is a big problem with several causes and big consequences to the global ecosystem as mentioned in the introduction of this chapter. This comes to account due one of the next work involving the presented heuristic algorithm for drone swarm auto-organization is to monitor forests to detect illegal tree cutting. Not only surveying forests with the flying unmanned aerial vehicles (UAVs), but equip this swarm agents with image recognition based on a machine learning model to be developed. The authors of this chapter are now starting to get involved on this endeavor, as depicted in figure 3.

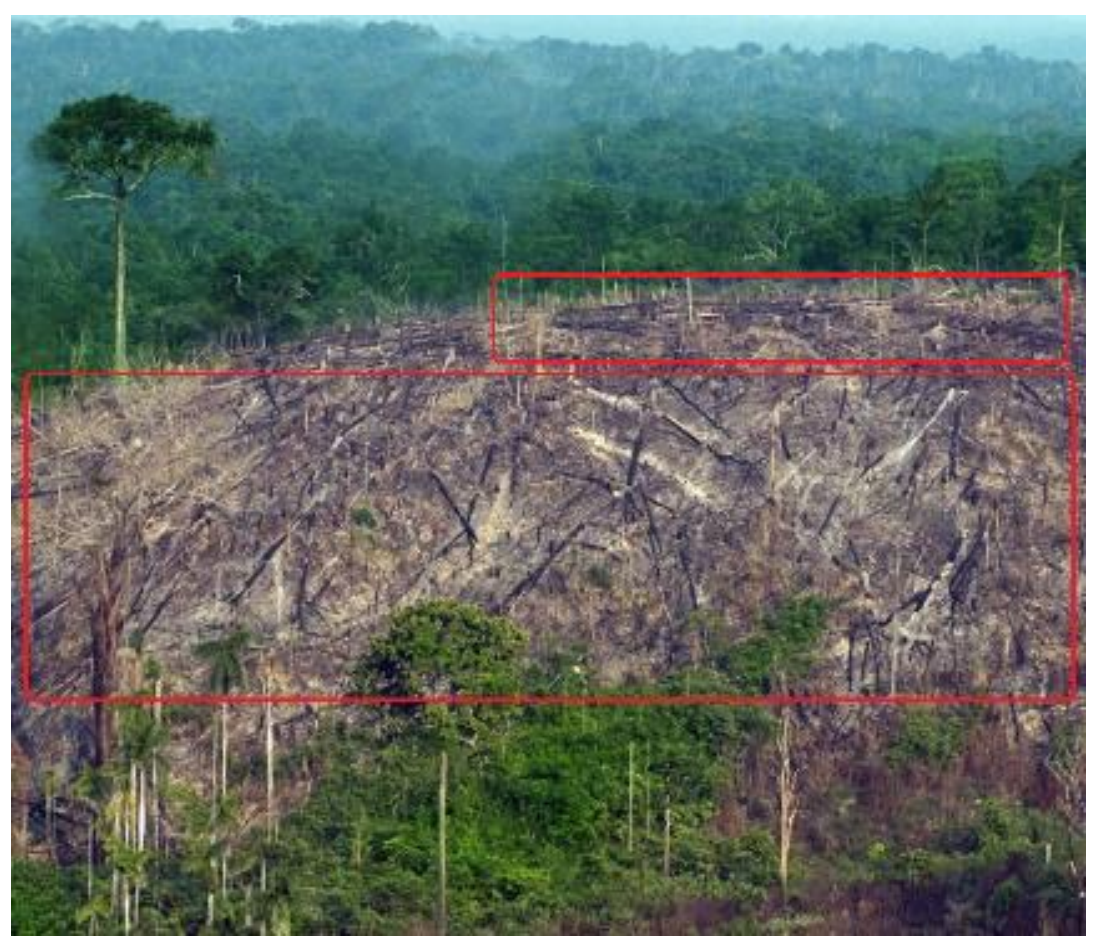

Fig. 3. A wood portion with illegal tree cutting identified by a machine learning model.

\section{$5 \quad$ Conclusions and Future Research}

The investigation has proven to be functional to adequately detect the beginning of a fire and how to give notice to the corresponding authorities. The relevance of our study lies in being able to identify the dimensionality (size of the fire). In our future work, the propagation of a fire will be analyzed by Support Vector Machine, analyzing the factors of the climate as in $[3,4,5]$.

Additionally, using the artificial deep learning intelligence technique, we are looking to identify recurring patterns in the beginning of the fire on the forest in the southwest 
of Chihuahua and how to determine the amount of trees that must be had to properly reforest after a large magnitude fire has occurred.

\section{References}

1. Malhi, Y., Roberts, J.T., Betts, R.A., Killeen, T.J., Li, W., Nobre, C.A.: Climate change, deforestation, and the fate of the Amazon. Science, 319(5860), pp. 169-172 (2008)

2. CNF: http://www.cnf.gob.mx:8090/snif/seif_chihuahua/programas/combatedeincendiosprevencion (2017)

3. Yanmaz, E., Yahyanejad, S., Rinner, B., Hellwagner, H., Bettstetter, C.: Drone networks: Communications, coordination, and sensing. Ad Hoc Networks, 68, pp. 1-15 (2018)

4. Colorado, J., Pérez, M.G., Mondragón, I., Méndez, D., Parra, C., Devia, C., Martinez, J.J., Molina, L. N.: An integrated aerial system for landmine detection: SDR-based Ground Penetrating Radar onboard an autonomous drone. Advanced Robotics, 31(15), pp. 791808 (2017)

5. X. Wang, S. Poikonen, B.L.: Golden: The vehicle routing problem with drones: several worst-case results. Optimization Letters ,11(4), pp. 679-697 (2017) 